\title{
Clinical and economic burden of neovascular age-related macular degeneration by disease status: a US claims-based analysis
}

\author{
Arghavan Almony, MD; Katelyn R Keyloun, PharmD, MS; Bijal Shah-Manek, BPharm, PhD; Jasjit K Multani, MPH; \\ Catherine B McGuiness, MA, MSc; Chi-Chang Chen, PhD, MsPharm; and Joanna H Campbell, PhD
}

\section{What is already known about this subject}

- Neovascular age-related macular degeneration (nAMD) accounts for approximately $90 \%$ of vision loss attributed to age-related macular degeneration (AMD), a leading cause of blindness in the United States.

- Previous studies suggest a substantial financial burden in nAMD but have been mostly focused on the Medicare population.

- Information on the clinical and economic burden among patients with $\mathrm{nAMD}$, overall and by disease status, is limited.

\section{What this study adds}

- This study evaluates the burden of nAMD disease in the US commercially insured population by baseline disease status and laterality.

- Patients with active choroidal neovascularization (CNV) incurred at least $\$ 7,000$ higher all-cause annual costs than patients with inactive CNV or inactive scar, largely driven by higher outpatient/anti-vascular endothelial growth factor (anti-VEGF) costs.

- Overall rates and frequency of antiVEGF treatment in this real-world commercially insured population remain lower than number of injections received in clinical trials.

\section{Author affiliations}

Arghavan Almony, MD, Carolina Eye Associates, Southern Pines, NC. Katelyn R Keyloun, PharmD, MS, Global Health Economics and Outcomes Research, Allergen, an AbbVie Company, Irvine, CA. Bijal Shah-Manek, BPharm, PhD, Global Health Economics and Outcomes Research, Noesis Healthcare Technologies, Inc, Redwood City, CA. Jasjit K Multani, MPH, Health Economics and Outcomes Research, Real-World Evidence, IQVIA Inc, Falls Church, VA. Catherine B McGuiness, MA, MSc, and Chi-Chang Chen, PhD, MsPharm, Health Economics and Outcomes Research, Real-World Evidence, IQVIA Inc, Plymouth Meeting, PA. Joanna H Campbell, PhD, Global Health Economics and Outcomes Research, Allergan, an AbbVie Company, Irvine, CA.

AUTHOR CORRESPONDENCE: Katelyn Keyloun, 714.246.5324; katelyn.keyloun@abbvie.com

J Manag Care Spec Pharm 2021;27(9):1260-72

Copyright $@ 2021$, Academy of Managed Care Pharmacy. All rights reserved.

OBJECTIVES: To examine the clinical and economic burden in patients with nAMD by disease status in the commercially insured US patient population and to identify drivers of nAMD-related costs.

METHODS: Patients with at least 1 International Classification of Diseases, 10th
Revision Clinical Modification (ICD-10-CM) diagnosis for $n A M D$ were identified from the IQVIA PharMetrics Plus database between April 2016 and August 2017 (index period). Patients had continuous enrollment for at least 6 months before and at least 12 months after the index date. Eye-level disease status 
was reported, along with intravitreal anti-VEGF treatment patterns. Health care resource utilization (HRU) (all-cause and nAMD-related) and direct health care costs were estimated over the 12 month follow-up period. Outcomes associated with falls and fractures were also assessed. Multivariate analysis identified drivers of annual nAMD-related outpatient costs among patients with anti-VEGF therapy. Incident patients (defined as those without an nAMD diagnosis 6 months prior to the index date) with at least 18 months of continuous enrollment after the index date were identified for a subset analysis to evaluate documented changes in disease status.

RESULTS: A total of 6,076 patients with nAMD were identified for the prevalent cohort; $60.1 \%, 17.2 \%$, and $5.9 \%$ had active CNV, inactive CNV, and inactive scar disease stage at index, respectively. The nAMD-related outpatient visit costs were roughly 4 and roughly 7 times higher, respectively, for the active CNV group $(\$ 8,658$ $[\mathrm{SD}=\$ 11,612])$ compared with the inactive CNV $(\$ 2,406[\mathrm{SD}=\$ 5,510])$ and inactive scar $(\$ 1,198[S D=\$ 3,035])$ groups $(P<0.0001)$. About $10 \%$ of prevalent patients had a fall/fracture claim over 12 months of follow-up. A total of 3,623 prevalent patients (59.6\%) were eligible for the anti-VEGF treatment patterns analysis (mean [SD] duration of therapy $=7.7$ [4.5] months; mean [SD] number of injections $=6.0$ [3.7]). Qualified incident cases comprised $17.8 \%(n=1,081)$ of the prevalent cohort. Approximately $20 \%$ of incident eyes with active CNV at baseline transitioned to inactive CNV. A total of 427 incident patients (39.5\%) qualified for anti-VEGF treatment patterns analysis (mean [SD] duration of therapy $=6.2$ [4.7] months, mean [SD] number of injections=5.2 [3.5]). Significant drivers of total nAMD-related costs were the initial anti-VEGF agent and anti-VEGF injection frequency $(P<0.0001)$ in both prevalent and incident cohorts.

CONCLUSIONS: The clinical and economic burden of nAMD treatment is substantial to the US healthcare system, where economic burden is higher among those with active CNV. Appropriate treatment may increase the duration of inactive disease periods and preserve visual acuity while lowering costs.

Age-related macular degeneration (AMD) is one of the leading causes of vision impairment and blindness in the United States, particularly in the elderly. ${ }^{1-5}$ In 2010, 2.1 million adults age 50 years and older in the US had AMD. ${ }^{6}$ Due to the aging population, this estimate is expected to reach 5.4 million by $2050 .{ }^{3}$ Although an estimated $80 \%$ of AMD cases are non-neovascular (or "dry"), ${ }^{7}$ the majority of vision impairment due to AMD is caused by neovascular AMD (nAMD; or "wet" AMD), characterized by choroidal neovascularization $(\mathrm{CNV}) .{ }^{8}$ Vision impairment due to nAMD is also associated with increased clinical burden, including depression, $, 9,10,12$ higher risk of falls/fractures,${ }^{11}$ and overall decreased quality of life. ${ }^{12}$ Declining visual acuity is also associated with decreasing quality of life. ${ }^{11}$ However, the relationship between disease stage, based on the activity of CNV, and the clinical and economic burden in nAMD is not well studied.

Estimates of the economic burden of nAMD in the United States are currently dated and largely focused on Medicare beneficiaries. Coleman et al reported higher all-cause median annual Medicare payments for patients with nAMD $(\$ 2,353)$ compared to patients with non-nAMD $(\$ 1,539)$ from 1995-199913; however, these estimates preceded intravitreal anti-vascular endothelial growth factor (anti-VEGF) use, which is now the standard of care, and changed the treatment landscape, allowing preservation of vision. ${ }^{14}$ Qualls et al reported all-cause mean annual Medicare payments of $\$ 12,422$ among beneficiaries with newly diagnosed nAMD. ${ }^{15}$ The authors also estimated AMDrelated Medicare payments and reported 50\% higher costs for patients diagnosed in 2008 than in 2004, which was largely due to higher pharmacy costs from the advent of anti-VEGF therapy. Cost estimates including commercially insured patients with nAMD are limited to those receiving anti-VEGF treatment. In a recent retrospective analysis of US claims data, total all-cause costs among anti-VEGF treatment-naïve and previously treated patients (roughly $60 \%$ commercially insured) with nAMD over 12 months were, on average, $\$ 21,125$ and $\$ 23,096$, respectively. ${ }^{16}$

Anti-VEGF therapy for nAMD places a burden on patients and health care system, as it requires multiple treatments that entail frequent office visits and ancillary testing. However, the frequency of anti-VEGF therapy over the first year in US clinical practice appears to be lower than the more frequent schedules used in randomized clinical trials $^{16-19}$ with discontinuation rates as high as $57 \%$ in the first year. ${ }^{19}$ This suggests potential undertreatment in patients with nAMD exists.

The overall burden of nAMD in commercially insured US patients is not well understood. Thus, the objectives of this study were to evaluate the clinical and economic burdens, including falls/fractures and anti-VEGF treatment patterns, of commercially insured patients in the US with nAMD aged at least 50 years, overall and by disease status.

\section{Methods}

\section{STUDY DESIGN}

This study was an observational, retrospective cohort study using US health insurance claims.

\section{DATA SOURCE}

Data extracted from the IQVIA PharMetrics Plus database from October 1, 2015, to August 31, 2018, (study period) was used for this study. The aggregated PharMetrics Plus 
database is a US health plans claims database comprised of fully adjudicated medical and pharmacy claims from more than 190 million unique enrollees (approximately 150 million at the time of the study $)^{20}$ and is representative of the national commercially insured population in terms of age and gender. The database is fully compliant with the Health Insurance Portability and Accountability Act of 1996 to maintain patient confidentiality. As such, no institutional review board approval was required.

\section{STUDY POPULATION}

Patients were required to meet the following criteria: (1) at least 1 nonancillary claim with an International Classification of Diseases, Tenth Revision, Clinical Modification (ICD10-CM) diagnosis code for nAMD (Supplementary Table 1, available in online article) during the April 1, 2016, through August 31, 2017, index period (date of the first diagnosis during this period served as the index date); (2) age of at least 50 years on the index date; (3) continuous health plan enrollment for at least 6 months before, and at least 12 months after, the index date; and (4) commercial insurance. Patients were excluded from the study if they had noncommercial insurance (eg, Medicaid, Medicare Advantage) or an unknown payer type on the index date.

Four patient subgroups, defined by nAMD disease status (active $\mathrm{CNV}$, inactive $\mathrm{CNV}$, inactive scar, and unspecified stage) on the index date, were identified for analysis. The nAMD disease status was determined based on the seventh digit of the ICD-10-CM code on the index date. For bilateral patients coded separately for the right and left eye, disease status from the more severe eye was assigned based on the following hierarchy: active $\mathrm{CNV}>$ inactive $\mathrm{CNV}>$ unspecified $>$ inactive scar. Patients were also stratified by laterality based on the seventh digit of the ICD-10-CM code (unilateral, bilateral, or unspecified). Patients with inconsistent disease status records on the index date were excluded from the study.

Subset of Patients With Newly Diagnosed nAMD (Incident Cohort). A subset of newly diagnosed patients was identified using a "washout period" (defined as the absence of an ICD-10-CM diagnosis code for nAMD during the 6-month pre-index period; Supplementary Figure 1, available in online article). This subset of patients was also required to have at least 18 months of follow-up in order to evaluate the documented change in disease status (eye-level transition from active CNV to inactive $\mathrm{CNV}$ ), in addition to their clinical and economic burden. The 18-month follow-up period was determined to be sufficient to evaluate this outcome based on clinical trials which used 1-2 years of follow-up. ${ }^{21-23}$

\section{OUTCOMES}

Baseline Demographic and Clinical Characteristics. Baseline demographic characteristics were measured at the index date and included age, gender, and US geographic region. Baseline clinical characteristics were measured over the 6-month pre-index (baseline) period or at index, unless otherwise specified. Diagnosis codes from the ICD-10-CM were used to identify general comorbid conditions of interest that included cardiovascular, metabolic, renal, hepatic, pulmonary, musculoskeletal, ocular, mental and central nervous system disorders. Charlson Comorbidity Index (CCI) scores were calculated according to the DartmouthManitoba adaptation. ${ }^{24}$ Diagnosis codes were used to identify claims for fall/fracture, which was an outcome of interest given the relationship between vision loss and fall/ fracture. Prior anti-VEGF use was identified using a combination of Healthcare Common Procedure Coding System (HCPCS) codes and National Drug Codes (NDC) for bevacizumab, ranibizumab, and aflibercept; proxy rules were used to identify intravitreal bevacizumab use for nAMD, as bevacizumab has multiple indications (Supplementary Table 2, available in online article).

Clinical Outcomes. The transition from active CNV to inactive CNV at the eye level was evaluated in the incident cohort over an 18-month follow-up period to assess a directional, clinically relevant, transition from active to inactive or quiescent CNV status. Time to the first observed, documented (ICD-10-CM diagnosis) transition from active CNV to inactive CNV during follow-up was reported.

The proportion of patients with a claim for fall/fracture over 6 and 12 months of follow-up was reported for the overall cohort (prevalent and incident) and by baseline disease status.

Healthcare Resource Utilization (HRU) and Cost. All-cause direct HRU and associated costs were estimated over the 12-month post-index period and reported by baseline disease status and laterality. Mean annual all-cause HRU was reported for the following resource categories: outpatient visits (physician and nonphysician office [eg, laboratory, ancillary care, and ambulatory surgeries] visits), emergency department [ED] visits, inpatient visits, and outpatient pharmacy utilization. Mean annual all-cause healthcare costs were reported as total health plan allowed costs (payer paid and patient copay) incurred and were broken into the following cost components: outpatient visits (physician and nonphysician office visits), ED visits, inpatient visits, and outpatient pharmacy utilization. Cost drivers of nAMD-related outpatient costs (post initial anti-VEGF treatment) were identified in covariate-adjusted analyses 


\section{FIGURE 1 Selection of Study Population (Attrition)}

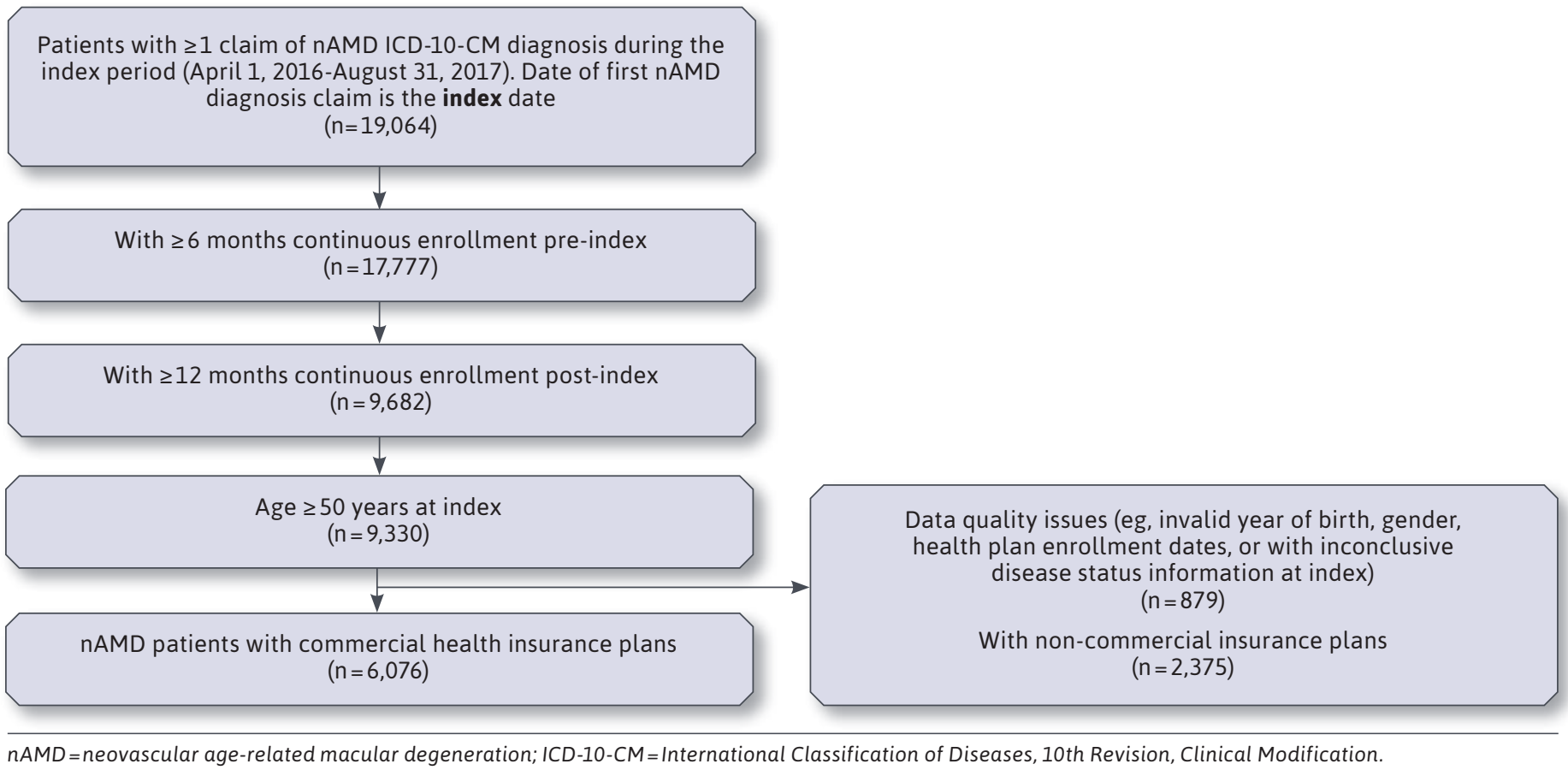

nAMD = neovascular age-related macular degeneration; ICD-10-CM = International Classification of Diseases, 10th Revision, Clinical Modification

among patients with anti-VEGF use in the prevalent and incident cohorts.

Total nAMD-related outpatient HRU (defined as at least 1 outpatient claim with an ICD-10-CM diagnosis code for nAMD in any position) and associated costs were also reported along with the following subcategories: (1) antiVEGF-related (ie, visits for anti-VEGF treatment [based on relevant HCPCS codes]) and (2) visits with optical coherence tomography testing (Current Procedural Terminology code 92134). Total annual per patient all-cause and component costs were reported on a per patient per year basis for the total cohort (ie, the denominator was the total cohort, regardless of whether the patient incurred costs for that component resource being reported). All costs were adjusted to 2018 US dollars using the medical care component of the Consumer Price Index.

To be eligible for the anti-VEGF treatment patterns analysis, patients were required to have anti-VEGF use over the 12-month follow-up period and with at least 12 months of continuous enrollment post initial anti-VEGF therapy. This patient subset was stratified by initial antiVEGF therapy (first observed bevacizumab, ranibizumab, or aflibercept therapy during the 12-month post-index period). Patients with greater than 1 type of anti-VEGF therapy on the date of the first treatment were excluded from the treatment patterns analysis. Duration of therapy (in months) and frequency of injections were reported for overall anti-VEGF treatment and the initial anti-VEGF therapy. Treatment disruption was defined as a greater than 18-week gap between visits with anti-VEGF treatment based on maximum reported treat and extend regimens of 16 weeks ${ }^{25,26}$ with 2 additional weeks as a grace period to capture treatment. The proportion of patients with a treatment disruption was reported for overall anti-VEGF use and by initial anti-VEGF therapy. Time to first switch (post-treatment disruption) of anti-VEGF therapy was reported in months for overall anti-VEGF use (defined as the time between anti-VEGF initiation and the start date of the first drug switch). Time to the first switch from the initial anti-VEGF therapy was defined as the time between the initial anti-VEGF therapy and the start date of the first drug switch after an observed treatment disruption of the initial therapy.

\section{STATISTICAL ANALYSES}

Descriptive statistics were used to characterize the study population. For tests of statistical significance of unadjusted 


\section{TABLE 1 Patient Demographics and Clinical Characteristics, Overall and by Baseline Disease Status and Laterality - Prevalent Cohort}

\begin{tabular}{|c|c|c|c|c|c|c|c|c|}
\hline \multirow[b]{2}{*}{ Characteristic $^{\mathrm{a}}$} & \multirow{2}{*}{$\begin{array}{c}\text { All } \\
\text { prevalent } \\
\text { nAMD } \\
\text { patients, } \\
\mathrm{N}=6,076 \\
(\mathbf{1 0 0 \% )}\end{array}$} & \multicolumn{4}{|c|}{ Baseline disease status } & \multicolumn{3}{|c|}{ Laterality } \\
\hline & & $\begin{array}{c}\text { Active } \\
\text { CNV, } \\
N=3,653 \\
(60.1 \%)\end{array}$ & $\begin{array}{c}\text { Inactive } \\
\text { CNV, } \\
N=1,046 \\
(17.2 \%)\end{array}$ & $\begin{array}{c}\text { Inactive } \\
\text { scar, } \\
N=358 \\
(5.9 \%)\end{array}$ & $\begin{array}{c}\text { Stage } \\
\text { unspecified, } \\
\mathrm{N}=1,019 \\
(16.8 \%)\end{array}$ & $\begin{array}{c}\text { Unilateral, } \\
\mathrm{N}=4,540 \\
(\mathbf{7 4 . 7 \% )}\end{array}$ & $\begin{array}{c}\text { Bilateral, } \\
\mathrm{N}=1,423 \\
(23.4 \%)\end{array}$ & $\begin{array}{c}\text { Unspecified, } \\
\mathrm{N}=113 \\
(1.9 \%)\end{array}$ \\
\hline \multicolumn{9}{|l|}{ Age, years) } \\
\hline Mean (SD) & $68.8(10.1)$ & $68.8(10.0)$ & $68.2(10.1)$ & 70.1 (10.9) & $69.2(10.3)$ & $68.4(10.0)$ & $70.4(10.4)$ & $66.6(10.3)$ \\
\hline Median & 66.0 & 66.0 & 65.0 & 67.0 & 67.0 & 65.5 & 70.0 & 63.0 \\
\hline \multicolumn{9}{|l|}{ Age Group, n (\%) } \\
\hline $50-54$ years & $336(5.5)$ & $184(5.0)$ & $66(6.3)$ & $20(5.6)$ & $66(6.5)$ & $249(5.5)$ & $74(5.2)$ & $13(11.5)$ \\
\hline $55-59$ years & $851(14.0)$ & 515 (14.1) & $160(15.3)$ & $49(13.7)$ & $127(12.5)$ & $664(14.6)$ & 169 (11.9) & $18(15.9)$ \\
\hline $60-64$ years & $1,561(25.7)$ & $956(26.2)$ & $286(27.3)$ & $83(23.2)$ & $236(23.2)$ & $1,219(26.9)$ & $314(22.1)$ & $28(24.8)$ \\
\hline $65-69$ years & 724 (11.9) & 435 (11.9) & $109(10.4)$ & $44(12.3)$ & $136(13.3)$ & $557(12.3)$ & $153(10.8)$ & $14(12.4)$ \\
\hline 70-74 years & $562(9.2)$ & $354(9.7)$ & $95(9.1)$ & $17(4.7)$ & $96(9.4)$ & $418(9.2)$ & $133(9.3)$ & $11(9.7)$ \\
\hline$\geq 75$ years & $2,042(33.6)$ & $1,209(33.1)$ & $330(31.5)$ & $145(40.5)$ & $358(35.1)$ & $1,433(31.6)$ & $580(40.8)$ & $29(25.7)$ \\
\hline Female, n (\%) & $3,346(55.1)$ & $2,012(55.1)$ & $597(57.1)$ & 190 (53.1) & $547(53.7)$ & $2,461(54.2)$ & $821(57.7)$ & $64(56.6)$ \\
\hline \multicolumn{9}{|l|}{ Geographic Region, n (\%) } \\
\hline Northeast & $1,542(25.4)$ & $934(25.6)$ & $261(25.0)$ & 105 (29.3) & $242(23.7)$ & $1,122(24.7)$ & 383 (26.9) & $37(32.7)$ \\
\hline Midwest & $1,511(24.9)$ & $921(25.2)$ & $269(25.7)$ & $87(24.3)$ & $234(23.0)$ & $1,146(25.2)$ & $339(23.8)$ & $26(23)$ \\
\hline South & $1,913(31.5)$ & $1,183(32.4)$ & $359(34.3)$ & $108(30.2)$ & $263(25.8)$ & $1,436(31.6)$ & $452(31.8)$ & $25(22.1)$ \\
\hline West & $1,109(18.3)$ & $614(16.8)$ & $157(15.0)$ & $58(16.2)$ & $280(27.5)$ & 835 (18.4) & $249(17.5)$ & $25(22.1)$ \\
\hline Other/unknown & $1(0.02)$ & $1(0.0)$ & $0(0.0)$ & $0(0.0)$ & $0(0.0)$ & $1(0.0)$ & $0(0.0)$ & $37(32.7)$ \\
\hline Anti-VEGF use ${ }^{\mathrm{b}}, \mathrm{n}(\%)$ & $2,671(44.0)$ & $1,978(54.1)$ & $261(25.0)$ & $27(7.5)$ & $405(39.7)$ & $1,965(43.3)$ & $666(46.8)$ & $0(0)$ \\
\hline Bevacizumab & $1,344(22.1)$ & $998(27.3)$ & $141(13.5)$ & $17(4.7)$ & $188(18.4)$ & $317(7.0)$ & $125(8.8)$ & $40(35.4)$ \\
\hline Ranibizumab & $449(7.4)$ & $351(9.6)$ & $36(3.4)$ & $3(0.8)$ & $59(5.8)$ & $1,002(22.1)$ & $327(23.0)$ & $7(6.2)$ \\
\hline Aflibercept & $1,102(18.1)$ & $798(21.8)$ & $96(9.2)$ & $7(2.0)$ & $20(19.7)$ & $787(17.3)$ & $289(20.3)$ & $15(13.3)$ \\
\hline History of falls/fractures, $n$ (\%) & $327(5.4)$ & $184(5.0)$ & $64(6.1)$ & $24(6.7)$ & $55(5.4)$ & $227(5.0)$ & $92(6.5)$ & $8(7.1)$ \\
\hline CCI Score, mean (SD) & $1.18(1.64)$ & $1.15(1.59)$ & $1.15(1.67)$ & $1.27(1.63)$ & $1.28(1.77)$ & $1.13(1.61)$ & $1.33(1.71)$ & $1.27(1.75)$ \\
\hline
\end{tabular}

continued on next page

HRU/costs, analysis of variance (for normally distributed variables) or Wilcoxon rank sum tests (for variables that are not normally distributed) were used to evaluate differences among continuous measures. A generalized linear model (GLM) was used to identify determinants of total annual nAMD-related outpatient costs post initial anti-VEGF treatment among patients with anti-VEGF use in the prevalent and incident cohorts. Covariates included baseline disease status, any documented transition in disease status at the eye level, documented change in laterality, initial anti-VEGF therapy, number of anti-VEGF injections, select baseline comorbidities, age, gender, and region. As such, patients with unspecified stage/laterality or unknown region were excluded from the adjusted cost analysis. Prior anti-VEGF use was tested in the prevalent cohort model only. Results of independent variable parameter estimates in the GLM were reported as exponentiated values with corresponding 95\% CIs. In the incident cohort, time from active CNV at index to first observed occurrence of inactive CNV was evaluated using Kaplan-Meier analysis. All analyses used SAS, version 9.4 (SAS Institute Inc., Cary, NC). 
TABLE 1 Patient Demographics and Clinical Characteristics, Overall and by Baseline Disease Status and Laterality - Prevalent Cohort (continued)

\begin{tabular}{|c|c|c|c|c|c|c|c|c|}
\hline \multirow[b]{2}{*}{ Characteristic $^{\mathrm{a}}$} & \multirow{2}{*}{$\begin{array}{c}\text { All } \\
\text { prevalent } \\
\text { nAMD } \\
\text { patients, } \\
\mathrm{N}=6,076 \\
(\mathbf{1 0 0 \% )}\end{array}$} & \multicolumn{4}{|c|}{ Baseline disease status } & \multicolumn{3}{|c|}{ Laterality } \\
\hline & & $\begin{array}{c}\text { Active } \\
\text { CNV, } \\
\mathrm{N}=3,653 \\
(60.1 \%)\end{array}$ & $\begin{array}{c}\text { Inactive } \\
\text { CNV, } \\
\mathrm{N}=1,046 \\
(17.2 \%)\end{array}$ & $\begin{array}{c}\text { Inactive } \\
\text { scar, } \\
\mathrm{N}=358 \\
(5.9 \%)\end{array}$ & $\begin{array}{c}\text { Stage } \\
\text { unspecified, } \\
\mathrm{N}=1,019 \\
(16.8 \%)\end{array}$ & $\begin{array}{l}\text { Unilateral, } \\
\mathrm{N}=4,540 \\
(\mathbf{7 4 . 7 \% )}\end{array}$ & $\begin{array}{c}\text { Bilateral, } \\
N=1,423 \\
(23.4 \%)\end{array}$ & $\begin{array}{c}\text { Unspecified, } \\
\mathrm{N}=113 \\
(1.9 \%)\end{array}$ \\
\hline \multicolumn{9}{|l|}{ Comorbidities: n (\%) } \\
\hline Anxiety/depression & $692(11.4)$ & $403(11.0)$ & $131(12.5)$ & $41(11.5)$ & $117(11.5)$ & $516(11.4)$ & $163(11.5)$ & $13(11.5)$ \\
\hline Cardiac arrhythmia & $835(13.7)$ & $478(13.1)$ & $150(14.3)$ & $59(16.5)$ & 148 (14.5) & $594(13.1)$ & $226(15.9)$ & $15(13.3)$ \\
\hline Diabetes & $1,356(22.3)$ & $806(22.1)$ & $213(20.4)$ & $79(22.1)$ & $258(25.3)$ & $991(21.8)$ & $328(23.0)$ & $37(32.7)$ \\
\hline Dyslipidemia & $2,533(41.7)$ & $1,493(40.9)$ & $443(42.4)$ & $141(39.4)$ & $456(44.7)$ & $1,896(41.8)$ & $591(41.5)$ & $46(40.7)$ \\
\hline Hypertension & $3,055(50.3)$ & $1,793(49.1)$ & $539(51.5)$ & $189(52.8)$ & $534(52.4)$ & $2,253(49.6)$ & $749(52.6)$ & $53(46.9)$ \\
\hline $\mathrm{MI} / \mathrm{CAD}$ & $784(12.9)$ & $460(12.6)$ & $131(12.5)$ & $51(14.2)$ & $142(13.9)$ & $589(13.0)$ & 179 (12.6) & $16(14.2)$ \\
\hline Osteoarthritis & $1,803(29.7)$ & $1,086(29.7)$ & $315(30.1)$ & $106(29.6)$ & $296(29.0)$ & $1,302(28.7)$ & $473(33.2)$ & $28(24.8)$ \\
\hline Sleep disorders & $676(11.1)$ & $404(11.1)$ & $131(12.5)$ & $30(8.4)$ & 111 (10.9) & $506(11.1)$ & $156(11.0)$ & $14(12.4)$ \\
\hline Thyroid disease & $836(13.8)$ & 473 (12.9) & 149 (14.2) & $58(16.2)$ & $156(15.3)$ & 609 (13.4) & $214(15.0)$ & $13(11.5)$ \\
\hline \multicolumn{9}{|l|}{ Ocular comorbidities, n (\%) } \\
\hline Diabetic retinopathy & $307(5.1)$ & $188(5.1)$ & $40(3.8)$ & $11(3.1)$ & $68(6.7)$ & $231(5.1)$ & $68(4.8)$ & $8(7.1)$ \\
\hline OAG/OHT & $445(7.3)$ & $233(6.4)$ & $87(8.3)$ & $38(10.6)$ & $87(8.5)$ & $324(7.1)$ & $109(7.7)$ & $12(10.6)$ \\
\hline Uveitis & $69(1.1)$ & $48(1.3)$ & $7(0.7)$ & $2(0.6)$ & $12(1.2)$ & 49 (1.1) & $17(1.2)$ & $3(2.7)$ \\
\hline OSD & $469(7.7)$ & $285(7.8)$ & $69(6.6)$ & $29(8.1)$ & $86(8.4)$ & $325(7.2)$ & $135(9.5)$ & $9(8)$ \\
\hline
\end{tabular}

${ }^{a}$ All characteristics were measured during the 6-month pre-index period or on index, except for prior anti-VEGF use (measured during 6-month pre-index period only).

${ }^{b}$ Not mutually exclusive.

$C A D=$ coronary artery disease; $C C I=$ Charlson Comorbidity Index; $C N V=$ choroidal neovascularization; $M I=$ myocardial infarction; $n A M D=$ neovascular age-related macular degeneration; $\mathrm{OAG}=$ open angle glaucoma; $\mathrm{OHT}=$ ocular hypertension; $\mathrm{OSD}=$ ocular surface disease.

\section{Results}

\section{PREVALENT COHORT}

A total of 6,076 patients with nAMD were included in the prevalent cohort (Figure 1); $60.1 \%(n=3,653), 17.2 \%(n=1,046)$, $5.9 \%(n=358)$, and $16.8 \%(n=1,019)$ had active $C N V$, inactive $\mathrm{CNV}$, inactive scar, and unspecified stage at index, respectively (Table 1). Most patients had unilateral disease (74.7\%) on index, and $1.9 \%(n=113)$ were of unknown laterality.

Baseline Patient Characteristics. The mean (SD) age of the prevalent cohort was 68.8 (10.1) years; most were female (Table 1). The most common comorbidities were hypertension, dyslipidemia, osteoarthritis, and diabetes. Overall, $7.7 \%$ of patients had ocular surface disease (OSD) and 5.4\% had a fall/fracture during the baseline period. About $44 \%$ of patients had prior anti-VEGF therapy with the highest rate observed for the active CNV group (54.1\%).
Clinical Outcomes. About 5.7\% $(n=344)$ of the prevalent cohort had a fall/fracture over 6 months post-index. The proportion of patients with a fall/fracture appeared to be highest for the inactive scar group $(n=25 ; 7.0 \%)$ compared with the active $\mathrm{CNV}(\mathrm{n}=193 ; 5.3 \%)$ and inactive $\mathrm{CNV}(\mathrm{n}=60$; $5.7 \%)$ groups. Over 12 months of follow-up, the rate was $10.4 \%(n=630)$ with similar trends seen among the 3 disease status groups.

Economic Burden. HRU data are presented in Table 2. Mean (SD) annual nAMD-related outpatient burden was 6.6 (4.8) visits overall; patients with active CNV had the highest utilization compared with patients with inactive $\mathrm{CNV}$ or inactive scar $(\mathrm{P}<0.0001)$. Overall mean $(\mathrm{SD})$ annual anti-VEGFrelated outpatient visit burden was 3.9 (4.2) visits, with the highest utilization seen in active CNV group compared with inactive $\mathrm{CNV}$ and inactive scar groups $(\mathrm{P}<0.0001)$. No statistically significant differences were observed in the proportion of patients with all-cause pharmacy utilization 


\section{TABLE 2 Health Care Resource Utilization Over the 12-Month Post-Index Perioda, Overall and by Baseline Disease Status-Prevalent Cohort}

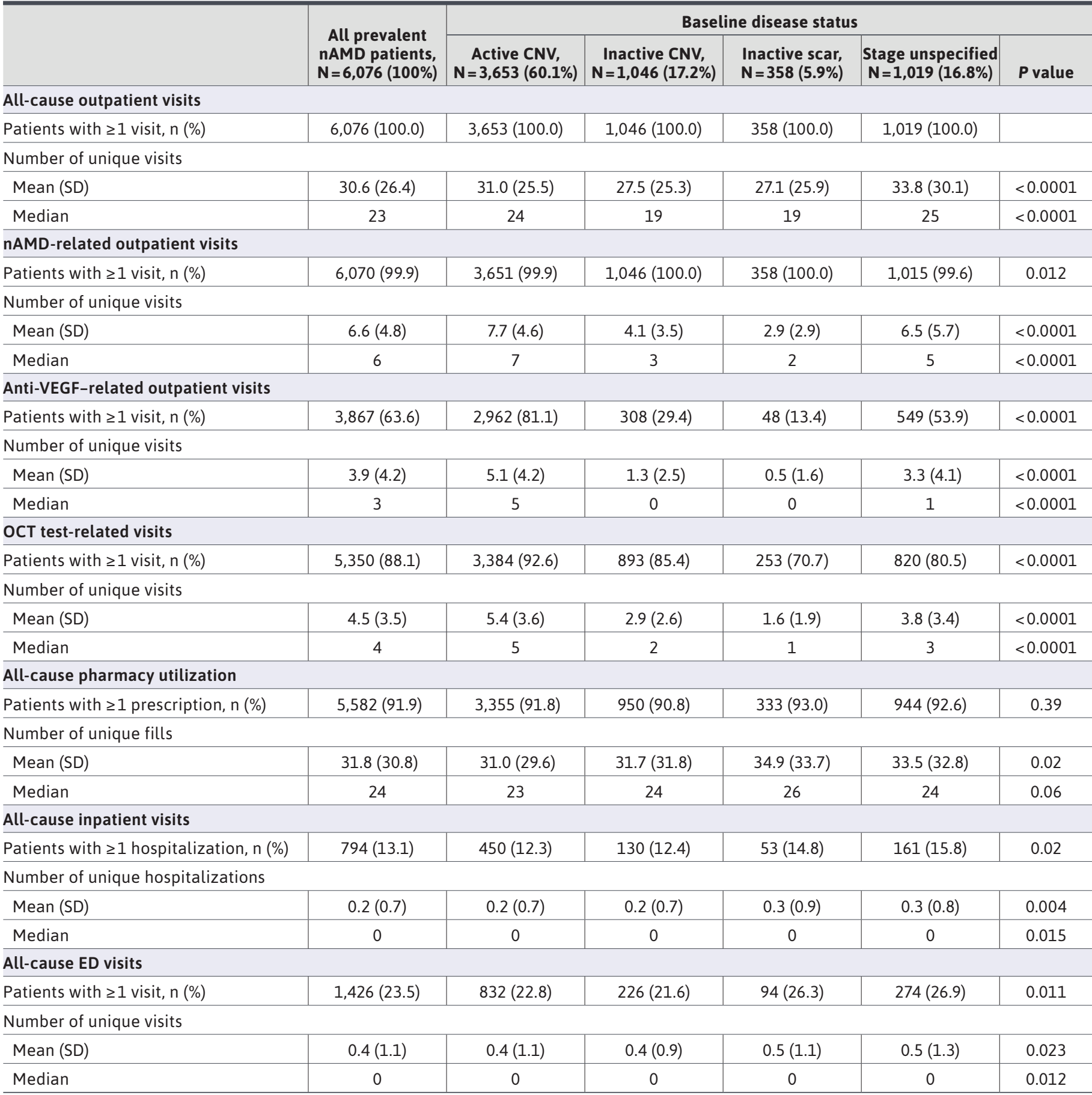

ancludes index date

$C N V=$ choroidal neovascularization; $E D=$ emergency department; $H R U=$ healthcare resource utilization; $n A M D=$ neovascular age-related macular degeneration; $\mathrm{OCT}=$ optical coherence tomography; $S \mathrm{~S}=$ standard deviation; $\mathrm{VEGF}=$ vascular endothelial growth factor. 


\section{FIGURE 2 Mean (SD) Annual nAMD-Related Outpatient Costs (2018 USD), Overall and by Baseline Disease} Status and Laterality-Prevalent Cohort

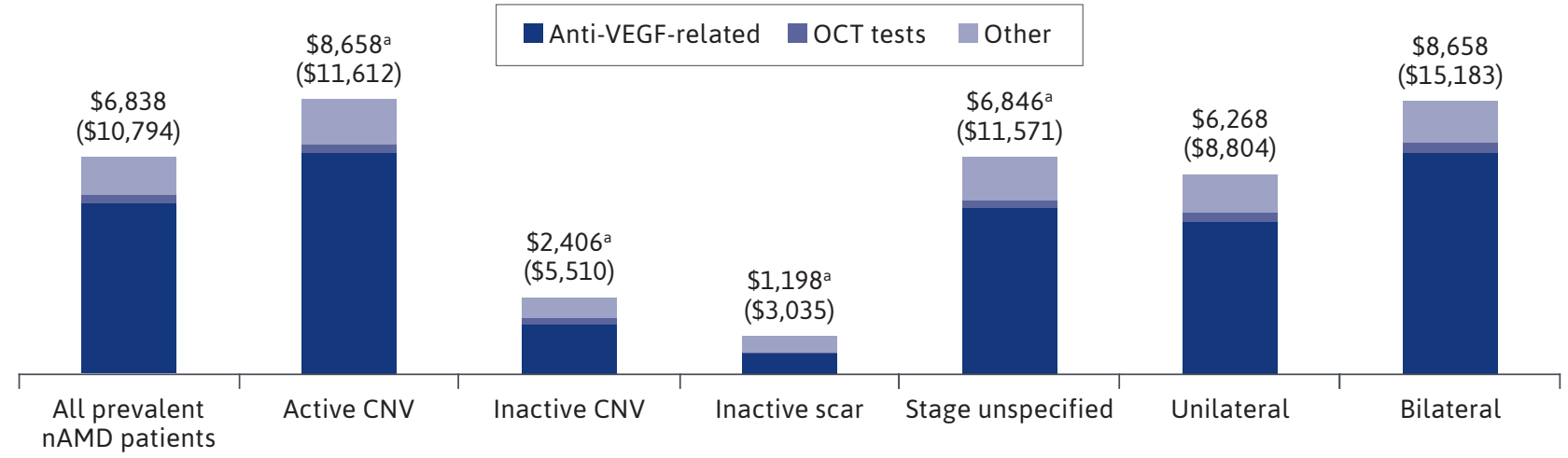

${ }^{a} \mathrm{P}<0.0001$ among all disease status groups

$\mathrm{CNV}=$ choroidal neovascularization; $\mathrm{nAM}=$ neovascular age-related macular degeneration; USD =US dollar.

by disease status, although the inactive scar group had the highest mean number of unique fills $(34.9, \mathrm{SD}=33.7)$. Mean (SD) number of hospitalizations and ED visits were highest in the inactive scar group compared with active CNV and inactive CNV groups. HRU data by baseline laterality are reported in Supplementary Table 3, available in online article.

Mean (SD) annual total all-cause cost for the prevalent cohort was $\$ 24,520(\$ 43,950)$ with outpatient costs as the largest cost component (63.5\%; Supplementary Figure 2, available in online article). The nAMD-related outpatient costs represented $28 \%$ of total costs and $44 \%$ of total outpatient costs, respectively, and were driven primarily by anti-VEGF injection-related costs $(\$ 5,357, \mathrm{SD}=\$ 9,380$; Figure 2). The nAMD-related outpatient visit costs were roughly 4 and roughly 7 times higher, respectively, for the active $\mathrm{CNV}$ group compared with the inactive $\mathrm{CNV}$ and inactive scar groups. The nAMD-related outpatient costs were numerically higher among bilateral patients compared to unilateral patients. Annual all-cause pharmacy costs were slightly higher for the active CNV group compared with the inactive CNV and inactive scar groups, though not statistically significant. Inpatient and ED costs were highest for inactive scar compared with active CNV and inactive CNV groups.

About $59.6 \%(n=3,623)$ of the prevalent cohort was eligible for the anti-VEGF treatment patterns analysis (Supplementary Table 4, available in online article). Among these patients, $29.6 \%$ received $1-3$ injections, $26.5 \%$ received 4-6 injections, and $43.9 \%$ received 7 or more injections over
1 year post initial treatment. Across agents, mean (SD) duration of therapy was 7.7 (4.5) months with a mean (SD) number of 6.0 (3.7) injections. Mean duration of therapy (SD) and mean (SD) number of injections was lowest for the bevacizumab group ( $\mathrm{n}=1,876$ [51.8\%]; 6.0 [4.6] months and 4.8 [3.3] injections, respectively) compared with the ranibizumab $(\mathrm{n}=539$ [14.9\%]; 7.6 [4.4] months and 6.0 [3.6] injections, respectively) and aflibercept groups $(n=1,208$ [33.3\%]; 7.9 [4.4] months and 6.1 [3.9] injections, respectively). Nearly half $(48.3 \%)$ of all patients $(n=1,750)$ had a treatment disruption. The proportion of patients who switched to a new anti-VEGF therapy from initial therapy was lowest in the aflibercept group (6.2\%) compared with the bevacizumab (15.8\%) and ranibizumab (14.3\%) groups, respectively.

Determinants of nAMD-Related Outpatient Costs Over 1-Year After Initial Anti-VEGF Treatment. A total of 3,103 prevalent anti-VEGF-treated nAMD patients of known disease status, laterality, and region at baseline were analyzed (Figure 3). Multivariate GLM results revealed an increasing trend in costs with increasing anti-VEGF injection frequency; there were $73 \%$ higher costs for patients with 4-6 injections to $342 \%$ higher costs for those with 10 or more injections compared with those with 1-3 injections over 1 year of follow-up $(\mathrm{P}<0.0001)$. Patients with ranibizumab or aflibercept as the initial therapy had higher costs compared with those with bevacizumab (268\% and 272\% higher, respectively; both $\mathrm{P}<0.0001)$. Baseline active CNV (ICD-10 code-based disease status measure) was associated with $6 \%$ higher cost compared with inactive $\mathrm{CNV} /$ scar patients; 


\section{FIGURE 3 Impact of Baseline Disease Status on nAMD-Related Outpatient Costs Among Patients With} Intravitreal Anti-VEGF Therapy, Prevalent Cohort $(\mathrm{N}=3,103)$

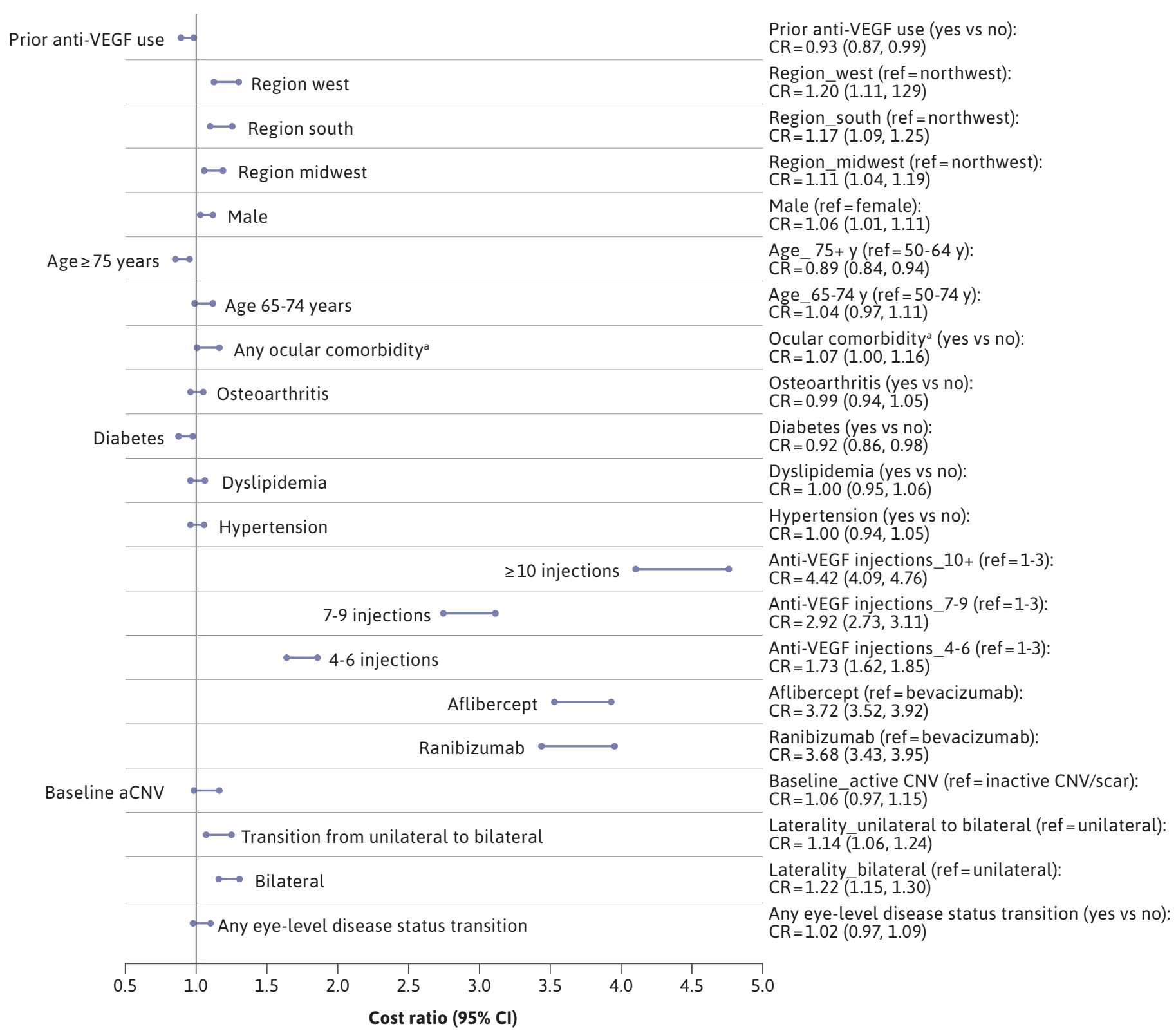


however the difference was not statistically significant. Patients with disease status transition, although not statistically significant, also trended toward having slightly higher annual cost (2\% higher). Bilateral patients and patients who transitioned from unilateral to bilateral had higher costs compared with patients who remained unilateral $22 \%$ and $14 \%$ higher, respectively; $\mathrm{P}<0.01)$. Other significant cost drivers were male sex $(6 \%$ higher; $\mathrm{P}<0.05)$ and patients located in the midwest, south or west (11\% to 20\% higher) compared with the northeast (all $\mathrm{P}<0.01$ ). Patients aged at least 75 years $(11 \%$ lower; $P<0.0001)$ or with diabetes $(8 \%$ lower; $\mathrm{P}<0.01$ ) were associated with lower costs compared with their reference groups.

\section{INCIDENT COHORT}

A total of 1,081 patients with newly diagnosed nAMD were identified, of which 46.3\% ( $n=501), 23.2 \% \quad(n=251), 11.5 \%$ $(\mathrm{n}=124)$, and $19.0 \%(\mathrm{n}=205)$ had active CNV, inactive CNV, inactive scar, and unspecified stage on index, respectively (Supplementary Table 5, available in online article). Most patients had unilateral disease $(77.2 \%)$, and $2.7 \%(n=29)$ were of unknown laterality. Baseline demographic characteristics were similar to the prevalent cohort.

Active CNV was found in $27.1 \%(n=570$ of 2104) of all eyes at baseline and among these, 28.1\% $(n=160)$ had a documented disease status transition over the 18-month follow-up period. Among eyes presenting with active CNV, $19.8 \%(n=113)$ transitioned to inactive CNV with a mean (SD) and median transition time of 7.7 (4.6) and 6.6 months, respectively. In eyes presenting with inactive CNV at baseline, $16.9 \%(n=54$ of 320$)$ subsequently transitioned back to active CNV. Approximately $10 \%(n=92)$ of incident patients had a fall/fracture over the 12-month follow-up period, where the active CNV group (9.4\%) appeared to have the highest rate of fall/fracture compared with inactive $\mathrm{CNV}$ (8.0\%) and inactive scar (7.3\%) groups.

Approximately 39.5\% $(n=427)$ of the incident cohort was eligible for the anti-VEGF treatment patterns analysis (Supplementary Table 6, available in online article). More patients in the incident cohort received 1-3 injections compared with the prevalent cohort over 12 months post initial anti-VEGF treatment (40.5\% vs $29.6 \%$ ); $21.3 \%$ received $4-6$ injections, and $38.2 \%$ received 7 or more injections. Mean (SD) duration of therapy was slightly lower vs the prevalent cohort (6.2 [4.7] vs 7.7 [4.5] months, respectively) with a mean (SD) number of 5.2 (3.5) injections (vs 6.0 injections in the prevalent cohort). The duration of therapy (mean [SD]) and mean number of injections was lowest for bevacizumab group in this subset as well $(\mathrm{n}=303$ [71.0\%]; 4.8 [4.4] months; 4.2 injections) vs ranibizumab ( $\mathrm{n}=40$ [9.4\%]; 6.4 [4.7] months; 5.9 injections) and aflibercept groups $(\mathrm{n}=84$ [19.7\%]; 6.6 [4.5] months; 5.1 injections). Nearly two-thirds of patients had a treatment disruption, which is higher than observed for the overall prevalent cohort ( $66 \%$ vs $48 \%$, respectively). The rate of switch was lowest in the aflibercept group (4.8\%) compared with bevacizumab (18.8\%) or ranibizumab (12.5\%) groups in the incident cohort.

Annual all-cause cost was lower for the incident cohort compared to the prevalent cohort (mean $[\mathrm{SD}]=\$ 18,943$ $[\$ 29,236]$ vs mean $[\mathrm{SD}]=\$ 24,520[\$ 43,950]$, respectively). The nAMD-related outpatient costs (mean $[\mathrm{SD}]=\$ 3,288$ [\$6,408]) represented almost one-third of all outpatient costs (mean $[\mathrm{SD}]=\$ 10,966[\$ 15,302])$. The nAMD-related outpatient cost was higher (at least 5-fold) for the active CNV group (mean $[\mathrm{SD}]=\$ 5,287[\$ 8,028]$ ) compared with inactive $\mathrm{CNV}$ (mean $[\mathrm{SD}]=\$ 985[\$ 2,613]$ ) and inactive scar (mean $[S D]=\$ 814[\$ 2,183])$ groups $(P<0.0001)$; costs were similar between unilateral and bilateral patients. Multivariate GLM results for the incident cohort revealed an increasing trend in nAMD-related outpatient costs with an increasing antiVEGF injection frequency $(102 \%$ higher costs for patients with 4-6 injections to $439 \%$ higher costs for those with 10 or more injections compared with those with 1-3 injections over 1 year of follow-up; $\mathrm{P}<0.0001)$ and higher costs among patients with ranibizumab or aflibercept as the initial anti-VEGF therapy compared with those with bevacizumab (203\% and 249\% higher, respectively; P<0.0001) (Supplementary Figure 3, available in online article).

\section{Discussion}

Prior studies reporting US cost estimates for nAMD have focused on the Medicare population ${ }^{27}$ and have supported the substantial financial burden of nAMD. ${ }^{28-32}$ Using the more detailed ICD-10-CM disease-coding system, our analysis provides an important update and focus on the economic burden of nAMD in the commercially insured US population by key clinical factors (disease status and laterality), as well as an assessment of cost drivers and clinical outcomes.

We estimated an annual all-cause cost for patients with nAMD of $\$ 24,520$ per patient, whereas newly diagnosed patients incurred slightly lower costs $(\$ 18,943)$. While not directly comparable methodologically (the sample used in the current study included patients with and without antiVEGF treatment), our cost estimates are similar to those reported by Kiss et al using a US administrative claims database. ${ }^{16}$ However, the current study is the first to report cost estimates by disease status, based on ICD-10-CM coding, which showed patients with active CNV had at least 4 times higher annual all-cause costs compared with inactive $\mathrm{CNV}$ or inactive scar. 
Results from adjusted analyses showed an increasing trend in nAMD-related costs with an increasing number of anti-VEGF injections in both prevalent and incident cohorts. This was expected because the major component of nAMD-related cost was anti-VEGF treatment in our study. Non-bevacizumab initial anti-VEGF therapy was associated with higher nAMD-related costs, which was most likely due to higher reimbursements for these 2 treatments, compared to bevacizumab. In the prevalent cohort, patients with bilateral disease and those who transitioned from unilateral to bilateral had up to $22 \%$ higher nAMDrelated outpatient costs compared to unilateral patients; this was possibly due to higher utilization of nAMD-related services (eg, anti-VEGF therapy and assessments). A similar trend was observed in the incident cohort; however, effect sizes were not statistically significant. Baseline active $\mathrm{CNV}$ and disease transition were both associated with directionally higher outpatient costs, although they were not of statistical significance in this analysis. As this claims analysis relied on observed ICD-10-CM coding for disease status, future studies with data on actual clinical disease activity such as from optical coherence tomography may be warranted to further evaluate its role in nAMD costs.

Bevacizumab was the most common initial anti-VEGF therapy observed overall. This is consistent with results from the 2018 American Society of Retina Specialists Preferences and Trends Surveys that report $70 \%$ of US specialists prefer bevacizumab over ranibizumab or aflibercept..$^{33}$ First-year anti-VEGF injection frequency in the incident cohort is similar to previous estimates of real-world anti-VEGF treatment patterns among treatment-naive patients with nAMD (mean of 5.6-6.0 injections in the first year). ${ }^{16,17}$ These realworld estimates are low when compared with randomized clinical trials that suggest at least 7-8 anti-VEGF injections are needed during the first year to gain meaningful change in visual acuity. ${ }^{34-36}$ A lower than optimal number of antiVEGF injections may lead to worse clinical outcomes and higher downstream costs. ${ }^{37-40}$ More importantly, as the number of anti-VEGF injections were found to be a primary cost driver in our study, our cost estimates may be lower than expected due to the suboptimal number of injections observed. Previous studies have evaluated the transition from active to inactive disease as a proxy for anti-VEGF effectiveness in the real-world setting. ${ }^{41,42}$ In our study, only $20 \%$ of incident nAMD eyes with active CNV at baseline had documented inactive CNV after 18 months of follow-up, further highlighting potential consequences of suboptimal number of anti-VEGF injections. A future analysis of eyes with anti-VEGF therapy or the transition from active to inactive disease status among patients may validate our findings.
The low-vision population is known to have a higher risk of falls/fractures. ${ }^{28-32}$ Prior observational studies have reported the estimated occurrence of fall/fractures among patients with nAMD in the preceding 12 months as high as $22 \%{ }^{26}$ (twice the odds compared to non-nAMD). ${ }^{11}$ These estimates were based on patient self-report of falls/fractures, including occurrences which did not require medical attention. In our study, we report a prevalence of approximately $10 \%$ of nAMD patients with a prior fall/fracture requiring medical attention as evidenced by health care insurance claims.

\section{LIMITATIONS}

Though the study provides a key insight into the economic burden of nAMD by baseline disease status, there are some limitations to our analysis. Because the study sample was composed of commercially insured patients, the findings are not generalizable to uninsured or non-commercially managed Medicare or Medicaid populations. This is especially relevant for nAMD since the disease is more prevalent in the elderly and results in vision impairment, potentially removing patients from the workforce-a key avenue for commercial insurance. The use of ICD-10-CM coding provides the ability to capture nAMD status and laterality from large databases. However, this information may be incomplete or not updated regularly; therefore, it may not adequately capture baseline disease status in all patients or fully capture post-index transitions. The results of this analysis may be confounded by "unobserved" factors (eg, important clinical parameters such as level of best corrected visual acuity and extent of visual field loss). It is possible that a small proportion of patients in our study sample had nAMD as a "rule-out" diagnosis based on our definition (at least 1 nonancillary claim with an ICD-10-CM diagnosis code for nAMD). Although we did not conduct a sensitivity analysis, potential misclassification of nAMD may have occurred. However, this definition is consistent with those used in prior real-world studies, ${ }^{17,45}$ and the distribution of nAMD-related outpatient visits over follow up from our study suggest this definition is specific. This study identified incident patients as a subset of the prevalent cohort. However, the washout period was only 6 months, which may not be a sufficient lookback period to identify incident cases of nAMD. Furthermore, the criteria to identify new cases of nAMD was based on the absence of diagnoses codes only; we did not exclude patients with prior use of therapies typically used to treat nAMD (eg, anti-VEGF) as those treatments are indicated for other retinal conditions, including diabetic macular edema and retinal vein occlusion, that can co-occur in patients developing nAMD. However, a small 
proportion of patients in the incident cohort had prior anti-VEGF use (2\%).

\section{Conclusions}

To our knowledge, this is the first study evaluating the clinical and economic burden of US commercially insured patients with nAMD by baseline disease status and laterality using the ICD-10-CM coding system. Findings show that the overall cost burden was highest among those with active CNV (vs inactive CNV or inactive scar) and with bilateral disease. Overall rates and frequency of treatment in this commercially insured population remains low compared to clinical trials. The type of anti-VEGF therapy and frequency of anti-VEGF injections were the most significant drivers of nAMD-related costs. Appropriate treatment may increase the duration of inactive disease periods and preserve visual acuity while lowering costs.

\section{DISCLOSURES}

This study was funded by Allergan, an AbbVie Company. Allergan employees were involved in the study design, interpretation of data, writing of the manuscript, and the decision to submit for publication. Keyloun and Campbell are employees of Allergan. Multani, McGuiness, and Chen are employees of IQVIA, which received funding from Allergan for conducting the analysis. Almony and Shah-Manek have nothing to disclose.

\section{ACKNOWLEDGMENTS}

The authors thank Wei-Ti Huang, manager of Data Science and Advanced Analytics at IQVIA, for data analysis and statistical support.

\section{REFERENCES}

1. Klein R, Klein BE, Linton KL. Prevalence of age-related maculopathy. The Beaver Dam Eye Study. Ophthalmology. 1992; 99(6):933-43. doi:10.1016/s0161-6420(92) 31871-8
2. Klaver CC, Wolfs RC, Vingerling JR, Hofman A, de Jong PT. Age-specific prevalence and causes of blindness and visual impairment in an older population: the Rotterdam Study. Arch Ophthalmol. 1998;116(5):653-58. doi:10.1001/ archopht.116.5.653

3. Congdon N, O'Colmain B, Klaver CCW et al. Causes and prevalence of visual impairment among adults in the United States. Arch Ophthalmol. 2004;122(4): 477-85. doi: 10.1001/archopht.122.4.477

4. Klein R, Peto T, Bird A, Vannewkirk MR. The epidemiology of age-related macular degeneration. Am J Ophthalmol. 2004;137(3):486-95. doi:10.1016/j. ajo.2003.11.069

5. Prevent Blindness America. Vision Problems in the US Prevalence of Adult Vision Impairment and Age-Related Eye Disease in America. 2008. Accessed February 10, 2020. http://preventblindness.org/wp-content/uploads/2013/04/ VPUS 2008 update.pdf

6. National Eye Institute. Age-Related Macular Dengeration (AMD) Tables. Updated February 7, 2020. Accessed February 7, 2020. https://www.nei. nih.gov/learn-about-eye-health/ resources-for-health-educators/ eye-health-data-and-statistics/age-relatedmacular-degeneration-amd-data-and-statistics/ age-related-macular-degeneration-amdtables

7. Kahn HA, Leibowitz HM, Ganley JP et al. The Framingham Eye Study. I. Outline and major prevalence findings. Am J Epidemiol. 1977;106(1):17-32. doi:10.1093/ oxfordjournals.aje.a112428

8. Ferris FL, Fine SL, Hyman L. Age-related macular degeneration and blindness due to neovascular maculopathy. Arch Ophthalmol. 1984;102(11):1640-42. doi:10.1001/ archopht.1984.01040031330019.

9. Brody BL, Gamst AC, Williams RA, et al. Depression, visual acuity, comorbidity, and disability associated with age-related macular degeneration. Ophthalmology. 2001;108(10):1893-900; discussion 1900-1. doi:10.1016/s0161-6420(01)00754-0
10. Rovner BW, Casten RJ, Tasman WS. Effect of depression on vision function in age-related macular degeneration. Arch Ophthalmol. 2002;120(8):1041-44. doi:10.1001/archopht.120.8.1041

11. Soubrane G, Cruess A, Lotery A, et al. Burden and health care resource utilization in neovascular age-related macular degeneration: findings of a multicountry study. Arch Ophthalmol. 2007;125(9): 1249-54. doi:10.1001/archopht.125.9.1249

12. Spooner KL, Mhlanga CT, Hong TH, Broadhead GK, Chang AA. The burden of neovascular age-related macular degeneration: a patient's perspective. Clin Ophthalmol. 2018;12:2483-91. doi:10.2147/ OPTH.S185052

13. Coleman AL, Yu F. Eye-related Medicare costs for patients with agerelated macular degeneration from 1995 to 1999. Ophthalmology. 2008;115(1):18-25. doi:10.1016/j.ophtha.2007.04.016

14. Flaxel CJ, Adelman RA, Bailey ST, et al. Age-Related Macular Degeneration Preferred Practice Pattern. Ophthalmology. 2020;127(1):P1-P65. doi:10.1016/j.ophtha.2019.09.024

15. Qualls LG, Hammill BG, Wang F, et al. Costs of newly diagnosed neovascular age-related macular degeneration among Medicare beneficiaries, 2004-2008. Retina. 2013;33(4):854-61. doi:10.1097/ IAE.0b013e31826f065e

16. Kiss S, Malangone-Monaco E, Wilson K, et al. Real-world injection frequency and cost of ranibizumab and aflibercept for the treatment of neovascular age-related macular degeneration and diabetic macular edema. J Manag Care Spec Pharm. 2020;26(3):253-66. doi:10.18553/jmcp.2020.19245

17. Kiss S, Campbell J, Almony A, et al. Management and outcomes for neovascular age-related macular degeneration: analysis of United States electronic health records. Ophthalmology. 2020;127(9): 1179-88. doi:10.1016/j.ophtha.2020.02.027

18. Johnston SS, Wilson K, Huang A, Smith D, Varker H, Turpcu A. Retrospective analysis of first-line anti-vascular endothelial growth factor treatment patterns in wet agerelated macular degeneration. Adv Ther. 2013;30(12):1111-27. doi:10.1007/ s12325-013-0078-4 
19. Lad EM, Hammill BG, Qualls LG, Wang F, Cousins SW, Curtis LH. AntiVEGF treatment patterns for neovascular age-related macular degeneration among Medicare beneficiaries. Am J Ophthalmol. 2014;158(3):537-43.e2. doi:10.1016/j. ajo.2014.05.014

20. IQVIA. US claims-IQVIA PharMetrics Plus. Published May 28, 2020. Accessed February 10, 2021. https://www. iqvia.com/library/ fact-sheets/ iqvia-pharmetrics-plus

21. Rosenfeld PJ, Brown DM, Heier JS, et al. Ranibizumab for neovascular agerelated macular degeneration. N Engl J Med. 2006;355(14):1419-31. doi:10.1056/ NEJMoa054481.

22. Brown DM, Kaiser PK, Michels M, et al. Ranibizumab versus verteporfin for neovascular age-related macular degeneration. N Engl J Med. 2006;355(14):1432-44. doi:10.1056/NEJMoa062655

23. Heier JS, Brown DM, Chong V, et al. Intravitreal aflibercept (VEGF trap-eye) in wet age-related macular degeneration. Ophthalmology. 2012;119(12):2537-48. Erratum in Ophthalmology. 2013;120(1): 209-10. doi:10.1016/j.ophtha.2012.09.006

24. Romano PS, Roos LL, Jollis JG. Adapting a clinical comorbidity index for use with ICD-9-CM administrative data: differing perspectives. J Clin Epidemiol. 1993;46(10):1075-79; discussion 1081-90. doi:10.1016/0895-4356(93)90103-8

25. Arnold JJ, Campain A, Barthelmes D, et al. Two-year outcomes of "treat and extend" intravitreal therapy for neovascular age-related macular degeneration. Ophthalmology. 2015;122(6):1212-19. doi:10.1016/j.ophtha.2015.02.009

26. DeCroos FC, Reed D, Adam MK, et al. Treat-and-extend therapy using aflibercept for neovascular age-related macular degeneration: a prospective clinical trial. Am J Ophthalmol. 2017;180:142-50. doi:10.1016/j.ajo.2017.06.002

27. Qualls LG, Hammill BG, Wang F, et al. Costs of newly diagnosed neovascular age-related macular degeneration among Medicare beneficiaries, 2004-2008.

Retina. 2013;33(4):854-61. doi:10.1097/ IAE.0b013e31826f065e
28. Cruess AF, Zlateva G, Xu X et al. Economic burden of bilateral neovascular age-related macular degeneration: multi-country observational study. Pharmacoeconomics. 2008;26(1):57-73.

29. Cruess A, Zlateva G, Xu X, Rochon S. Burden of illness of neovascular agerelated macular degeneration in Canada. Can J Ophthalmol. 2007 Dec;42(6):836-43.

30. Spooner KL, Mhlanga CT, Hong TH. The burden of neovascular age-related macular degeneration: a patient's perspective. Clin Ophthalmol. 2018 Dec 4;12:2483-91.

31. Soubrane G, Cruess A, Lotery A et al. Burden and health care resource utilization in neovascular age-related macular degeneration: findings of a multicountry study. Arch Ophthalmol. 2007 Sep;125(9):1249-54.

32. Ke KM. The direct, indirect and intangible costs of visual impairment caused by neovascular age-related macular degeneration. Eur J Health Econ. 2010 Dec;11(6):525-31.

33. HCPLive. ASRS Preferences and Trends Survey Documents State of AMD, Retina Care. 25 July 2018. Accessed 1 May 2020. Available at https://www.mdmag. com/conference-coverage/asrs-2018/ asrs-preferences-and-trends-survey-documents-state-of-amd-retina-care

34. Martin DF, Maguire MG, Fine SL, et al. Ranibizumab and bevacizumab for treatment of neovascular age-related macular degeneration: two-year results. Ophthalmology. 2012; 119(7):1388-98.

35. Rofagha S, Bhisitkul RB, Boyer DS, Sadda SR, Zhang K. Seven-year outcomes in ranibizumab-treated patients in ANCHOR, MARINA, and HORIZON: a multicenter cohort study (SEVEN-UP). Ophthalmology. 2013;120(11): 2292-99.

36. Singer MA, Awh CC, Sadda S, et al. HORIZON: an openlabel extension trial of ranibizumab for choroidal neovascularization secondary to age-related macular degeneration. Ophthalmology 2012;119(6):1175-83.
37. Wecker T, Grundel B, Reichl S, et al. Anti-VEGF injection frequency correlates with visual acuity outcomes in pro re nata neovascular AMD treatment. Sci Rep. 2019;9(1):3301.

38. Reich O, Bachmann LM, Faes L, et al. Anti-VEGF treatment patterns and associated health care costs in Switzerland: findings using real-world claims data. Risk Manag Healthc Policy. 2015;8:55-62.

39. Kataja M, Hujanen P, Huhtala $H$, Kaarniranta K, Tuulonen A, UusitaloJarvinen $\mathrm{H}$. Outcome of anti-vascular endothelial growth factor therapy for neovascular age-related macular degeneration in real-life setting. $\mathrm{Br} \mathrm{J}$ Ophthalmol. 2018;102(7):959-65.

40. Ciulla TA, Hussain RM, Pollack JS, Williams DF. Visual Acuity Outcomes and Anti-Vascular Endothelial Growth Factor Therapy Intensity in Neovascular AgeRelated Macular Degeneration Patients: A Real-World Analysis of 49485 Eyes. Ophthalmol Retina. 2020;4(1):19-30.

41. Silva-Garcia R, McLellan C, Shaya FS et al. Long-lasting effects of anti-VEGF/ photodynamic combination therapy in the treatment of exudative age-related macular degeneration: a retrospective chart review. Clin Ophthalmol. 2014 Dec 15;8:2529-32.

42. Almuhtaseb H, Kanavati S, Rufai SR et al. One-year real-world outcomes in patients receiving fixed-dosing aflibercept for neovascular age-related macular degeneration. Eye (Lond). 2017 Jun;31(6):878-83.

43. Coleman AL, Stone K, Ewing SK, et al. Higher risk of multiple falls among elderly women who lose visual acuity. Ophthalmology. 2004;111(5):857-62.

44. Harwood RH. Visual problems and falls. Age Ageing. 2001;30(suppl):13-18.

45. Day S, Acquah K, Lee PP, Mruthyunjaya P, Sloan FA. Medicare costs for neovascular age-related macular degeneration, 1994-2007. Am J Ophthalmol. 2011 Dec;152(6):1014-20. 